\title{
Study on Linear Vibration Model of Shield TBM Cutterhead Driving System
}

\author{
Xianhong Li, Haibin Yu, Peng Zeng and Mingzhe Yuan \\ Key Laboratory of Industrial Control Network and System, Shenyang Institute of Automation Chinese Academy of \\ Sciences, Shenyang, Liaoning province, China, 110016
}

\section{Jianda Han and Lanxiang Sun}

State Key Laboratory of Robotics, Shenyang Institute of Automation Chinese Academy of Sciences, Shenyang, Liaoning province, China, 110016

(Received 22 August 2012; provisionally accepted 23 October 2012; accepted 18 February 2013)

In this paper, a general linear time-varying multiple-axis (LTVMA) vibration model of shield tunnel boring machine (TBM) cutterhead driving system is established. The corresponding multiple inputs and multiple outputs (MIMO) state-space model is also presented. The linear vibration model is analysed, and the vibration-torque transfer function matrix and the vibration-torque static gain matrix are obtained. The linear vibration model is simulated, and the physical parameters' effects on the vibration response are investigated. A preliminary approach is proposed to reduce vibration by increasing motor rotor inertia and viscous damped. The LTVMA vibration model provides a solid foundation for fault detection and diagnosis (FDD), as long as the health monitoring of cutterhead driving system.

\section{NOMENCLATURE}

n The number of cutterhead driving motors;

q the reducer speed reduction ratio

$\mathbf{J}_{\mathbf{d}, \mathbf{i}} \quad \mathbf{i}$-th induction motor rotor inertia after equivalent coupling-i

$\mathbf{b}_{\mathbf{d}, \mathbf{i}} \quad \mathbf{i}$-th induction motor rotor viscous damped after equivalent coupling-i

$\mathbf{J}_{\mathbf{z}, \mathbf{i}} \quad$ Inertia of the $i$-th coupling between motor-i and reducer-i

$\mathbf{b}_{\mathbf{z}, \mathbf{i}} \quad$ Viscous damped of the $\mathbf{i}$-th coupling between motor-i and reducer-i

$\mathbf{J}_{\mathbf{r}, \mathbf{i}} \quad \mathbf{i}$-th induction motor rotor inertia

$\mathbf{b}_{\mathbf{r}, \mathbf{i}} \quad \mathbf{i}$-th induction motor rotor viscous damped

$\mathbf{J}_{\mathbf{m}} \quad$ Large gear inertia

$\mathbf{b}_{\mathbf{m}} \quad$ large gear viscous damped

$\mathbf{r}_{\mathbf{m}} \quad$ large gear radius

$\theta_{\mathbf{p}, \mathbf{i}} \quad$ Angular displacement of $\mathbf{i}$-th active pinion

$\theta_{\mathbf{i}} \quad$ angular displacement of $\mathbf{i}$-th motor rotor

$\theta_{\mathbf{m}} \quad$ Large gear angular displacement

$\omega_{\mathbf{m}} \quad$ large gear angular speed

$\mathbf{J}_{\mathbf{c}, \mathbf{i}} \quad \mathbf{i}$-th pinion inertia after equivlalent coupling-i

$\mathbf{b}_{\mathbf{c}, \mathbf{i}} \quad \mathbf{i}$-th pinion viscous damped after equivalent coupling-i

$\mathbf{J}_{\mathbf{w}, \mathbf{i}} \quad$ Inertia of the $\mathbf{i}$-th coupling between reduce-i and pinion-i

$\mathbf{b}_{\mathbf{w}, \mathbf{i}} \quad$ Viscous damped of the $\mathbf{i}$-th coupling between reducer-i and pinion-i

$\mathbf{J}_{\mathbf{p}, \mathbf{i}} \quad \mathbf{i}$-th pinion inertia;

$\mathbf{b}_{\mathbf{p}, \mathbf{i}} \quad \mathbf{i}$-th pinion viscous damped

$\mathbf{T}_{\mathbf{e}, \mathbf{i}} \quad \mathbf{i}$-th induction motor electrical magnetic torque

$\omega_{\mathbf{p}, \mathbf{i}} \quad$ Angular speed of pinion-i

$\omega_{\mathbf{i}} \quad$ angular speed of induction motor-i
$\mathbf{F}_{\mathbf{i}} \quad$ Elastic mesh force of the pinion-i and large gear

$\mathbf{r}_{\mathbf{i}} \quad$ radius of pinion-i

$\mathbf{M}_{\mathbf{c}, \mathbf{i}} \quad$ Elastic mesh torque of the pinion-i and large gear

$\mathbf{p}_{\mathbf{i}} \quad$ Relative position of the pinion-i and large gear

$\mathbf{k}_{\mathbf{i}} \quad$ Mesh stiffness of the pinion-i and large gear

$\mathbf{c}_{\mathbf{i}} \quad$ Mesh damped of the pinion-i and large gear

$\mathbf{m}_{\mathbf{p}, \mathbf{i}} \quad$ Mass of the pinion-i

$\mathbf{k}_{\mathbf{y}, \mathbf{i}} \quad$ support stiffness of pinion-i

$\mathbf{c}_{\mathbf{y}, \mathbf{i}} \quad$ support damped of pinion-i

$\mathbf{m}_{\mathrm{g}} \quad$ Mass of the large gear

$\mathbf{k}_{\mathbf{y}, \mathbf{m}} \quad$ support stiffness of large gear

$\mathbf{c}_{\mathbf{y}, \mathbf{m}} \quad$ support damped of large gear

$\mathbf{y}_{\mathbf{i}} \quad$ The contact direction (line-of-action direction) vibration displacement of $\mathbf{i}$-th pinion

$\mathbf{y}_{\mathbf{m}}^{\mathbf{i}} \quad$ The large gear's contact vibration displacement along i-th pinion's line-of-action direction

$\mathbf{T}_{\mathbf{L}} \quad$ Shield TBM cutterhead's load torque

$\mathbf{k}_{\mathbf{f}, \mathbf{i}}, \mathbf{c}_{\mathbf{f}, \mathbf{i}}$ elastic mesh force coefficient of pinion-i

$\mathbf{k}_{\mathbf{t}, \mathbf{i}}, \mathbf{c}_{\mathbf{t}, \mathbf{i}}$ elastic mesh torque coefficient of pinion-i

$\mathbf{i}_{\mathbf{m}, \mathbf{i}} \quad$ gear transmission ratio

$\mathbf{T}_{\mathbf{1}} \quad$ The resistant torque of soil and rocks

$\mathbf{T}_{2} \quad$ The friction torque of soil and rocks which chafe with the front of the cutterhead

$\mathbf{T}_{\mathbf{3}} \quad$ The friction torque of soil and rocks which chafe with the back of the cutterhead

$\mathbf{T}_{4} \quad$ The friction torque of soil and rocks which chafe with bulkhead

$\mathbf{T}_{\mathbf{5}} \quad$ The stirring torque of soil and rocks by cutterhead stirring rod

$\mathbf{T}_{6} \quad$ The friction torque of cutterhead bearings and sealed chamber 